\title{
TU/e emonown

\section{Heat-flux scaling in turbulent Rayleigh-Bénard convection with an imposed longitudinal wind}

\section{Citation for published version (APA):}

Scagliarini, A., Gylfason, A., \& Toschi, F. (2014). Heat-flux scaling in turbulent Rayleigh-Bénard convection with an imposed longitudinal wind. Physical Review E - Statistical, Nonlinear, and Soft Matter Physics, 89(4), 043012-1/5. [043012]. https://doi.org/10.1103/PhysRevE.89.043012

DOI:

10.1103/PhysRevE.89.043012

Document status and date:

Published: 01/01/2014

\section{Document Version:}

Publisher's PDF, also known as Version of Record (includes final page, issue and volume numbers)

\section{Please check the document version of this publication:}

- A submitted manuscript is the version of the article upon submission and before peer-review. There can be important differences between the submitted version and the official published version of record. People interested in the research are advised to contact the author for the final version of the publication, or visit the $\mathrm{DOI}$ to the publisher's website.

- The final author version and the galley proof are versions of the publication after peer review.

- The final published version features the final layout of the paper including the volume, issue and page numbers.

Link to publication

\section{General rights}

Copyright and moral rights for the publications made accessible in the public portal are retained by the authors and/or other copyright owners and it is a condition of accessing publications that users recognise and abide by the legal requirements associated with these rights.

- Users may download and print one copy of any publication from the public portal for the purpose of private study or research.

- You may not further distribute the material or use it for any profit-making activity or commercial gain

- You may freely distribute the URL identifying the publication in the public portal.

If the publication is distributed under the terms of Article $25 \mathrm{fa}$ of the Dutch Copyright Act, indicated by the "Taverne" license above, please follow below link for the End User Agreement:

www.tue.nl/taverne

Take down policy

If you believe that this document breaches copyright please contact us at:

openaccess@tue.nl

providing details and we will investigate your claim. 


\title{
Heat-flux scaling in turbulent Rayleigh-Bénard convection with an imposed longitudinal wind
}

\author{
Andrea Scagliarini, ${ }^{1,2,3}$ Ármann Gylfason, ${ }^{1}$ and Federico Toschi ${ }^{2,4,5}$ \\ ${ }^{1}$ School of Science and Engineering, Reykjavik University, Menntavegur 1, IS-101 Reykjavik, Iceland \\ ${ }^{2}$ Department of Mathematics and Computer Science, Eindhoven University of Technology, The Netherlands \\ ${ }^{3}$ Department of Physics and INFN, Univ. of Tor Vergata, Via della Ricerca Scientifica 1, 00133 Rome, Italy \\ ${ }^{4}$ Department of Applied Physics, Eindhoven University of Technology, The Netherlands \\ ${ }^{5}$ CNR-IAC, Via dei Taurini 19,00185 Rome, Italy \\ (Received 18 November 2013; published 11 April 2014)
}

\begin{abstract}
We present a numerical study of Rayleigh-Bénard convection disturbed by a longitudinal wind. Our results show that under the action of the wind, the vertical heat flux through the cell initially decreases, due to the mechanism of plume sweeping, and then increases again when turbulent forced convection dominates over the buoyancy. As a result, the Nusselt number is a nonmonotonic function of the shear Reynolds number. We provide simple models that capture with good accuracy all the dynamical regimes observed. We expect that our findings can lead the way to a more fundamental understanding of the complex interplay between mean wind and plume ejection in the Rayleigh-Bénard phenomenology.
\end{abstract}

DOI: 10.1103/PhysRevE.89.043012

PACS number(s): 47.27.-i, 47.55.pb

\section{INTRODUCTION}

Thermal convection plays an important role in many geophysical, environmental, and industrial flows, such as in Earth's mantle, in the atmosphere, and in the oceans, to name but a few relevant examples. In particular, the idealized case of Rayleigh-Bénard (RB) convection occurring in a layer of fluid confined between two differentially heated parallel plates under a constant gravitational field has been extensively studied [1-4]. However, in several real-life situations, the picture can be much more complex with horizontal winds perturbing natural convection. In the atmosphere, for instance, this competition plays a crucial role in the formation of thermoconvective storms [5]. On the other side, buoyancy effects can be relevant in a number of industrial processes based on forced convection, such as coiled heat exchangers [6]. Similarly, a combination of forced and natural convection is present in indoor ventilation applications [7-9].

According to the standard picture at the basis of the existing models for the scaling laws of the heat flux [10-13], the RB system is characterized by the multiscale coupling between large-scale circulation (mean wind) and detaching thermal structures from the boundary layers at the walls (plumes). Besides the above mentioned motivations, applying a mean wind to a natural convection setup can shed light on the effects of bulk flow on the boundary layer dynamics, thus helping to better understand one of the most intriguing feature of RB convection.

In this paper we report on a numerical study of RB convection with an imposed constant horizontal pressure gradient, orthogonal to gravity, that induces the wind (the so-called Poiseuille-Rayleigh-Bénard (PRB) flow setup [14-16]). We show that the heat transfer from the walls can be dominated by either the buoyancy or by the "forced" convection and that the interplay of the two mechanisms gives rise to a nontrivial dependence of the Nusselt number $\mathrm{Nu}$ on the parameter space that is spanned by the Rayleigh $\mathrm{Ra}$ and shear Reynolds numbers $\operatorname{Re}_{\tau}$ (quantifying, respectively, the intensity of buoyancy and of the pressure gradient relative to viscous forces).
Our main result consists of the observation that, taking a standard RB system as reference, Nu initially decreases (in agreement with numerical studies of RB convection with sheared boundary layers [17]) then, when the dynamics are completely dominated by the forced convection regime, it increases again with $\operatorname{Re}_{\tau}$. A phenomenological explanation for this behavior is provided together with discussions on the possible implications for the modeling of the $\mathrm{Nu}$ vs Ra relation in pure natural convection setup.

\section{DETAILS OF NUMERICAL METHOD}

The equations of motion for the fluid velocity $\mathbf{u}$ and temperature $T$ are

$$
\begin{gathered}
\partial_{t} \mathbf{u}+\mathbf{u} \cdot \nabla \mathbf{u}=-\frac{1}{\rho} \nabla P+v \nabla^{2} \mathbf{u}+\alpha \mathbf{g} T+\mathbf{f}, \\
\partial_{t} T+\mathbf{u} \cdot \nabla T=\kappa \nabla^{2} T,
\end{gathered}
$$

in addition to the incompressibility condition, $\nabla \cdot \mathbf{u}$. In our model, as in most turbulent RB and turbulent channel flow studies, we have omitted mechanical-to-internal energy conversion terms, which are negligible in turbulent flows (in our simulations, for instance, we measured heat generation by viscosity finding it to be at most about $1.5 \%$ of the total heat flux between the walls). The properties of the fluid are $\rho$ the fluid density (assumed constant), $v$ the kinematic viscosity, $\alpha$ the thermal expansion coefficient, and $\kappa$ the thermal diffusivity. $P$ is the pressure field, $\mathbf{g}=g \hat{z}$ is gravity, and $\mathbf{f}$ is a forcing term of the form $\mathbf{f}=(F / \rho) \hat{x} \equiv \tilde{F} \hat{x}$ ( $\hat{x}$ is the direction parallel to the walls or stream-wise direction). Equations (1) and (2) are evolved using a three-dimensional (3D) thermal lattice Boltzmann algorithm $[18,19]$ with two probability densities (for density and momentum and for temperature, respectively). This method has been extensively used to address numerically several important issues related to the physics of both thermal convection and turbulent channel flow [20,21]; in particular in Ref. [22] the code was validated and grid resolutions were tested against previous studies with different numerical methods (agreement was found both in the 

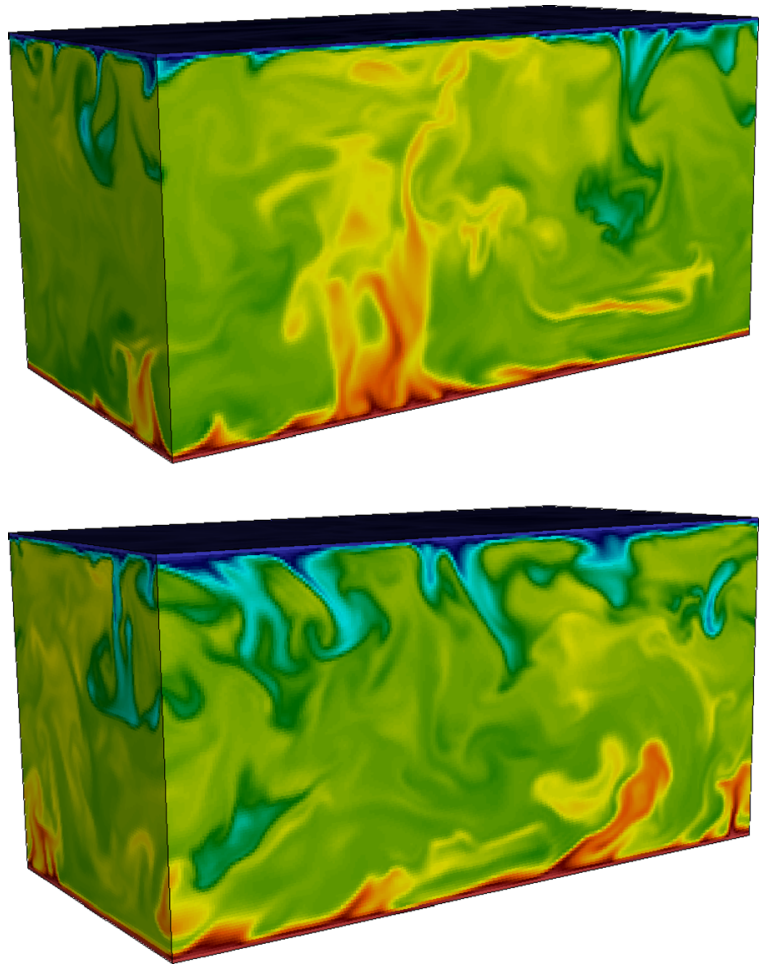

FIG. 1. (Color online) Snapshots of the temperature field for (top panel) the pure $\mathrm{RB}$ case $\left(\operatorname{Re}_{\tau}=0\right)$ at $\mathrm{Ra}=1.3 \times 10^{7}$. bottom: mixed convective case $\mathrm{Ra}=1.3 \times 10^{7}$ and $\mathrm{Re}_{\tau}=92$. In the pure-convective case, plumes are detaching from the bottom and top boundary layers and freely travel through the bulk to the opposite walls whereas, in the mixed case, considerable distortion of plumes occur due to the wind.

mean temperature profiles and in the values of $\mathrm{Nu}$ ). We adopt here a resolution of $256 \times 128 \times 128$ lattice points (see Fig. 1 for snapshots of the temperature field in the simulation cell) which provides a good accuracy [22] and, at the same time, makes the computational effort of spanning a two-dimensional parameter space affordable.

\section{RESULTS}

As mentioned in the introduction, to characterize the dynamics we need the Rayleigh number Ra, quantifying the strength of buoyancy (with respect to viscous forces),

$$
\mathrm{Ra}=\frac{\alpha g \Delta H^{3}}{\nu \kappa},
$$

where $\Delta=T_{\text {hot }}-T_{\text {cold }}$ is the temperature drop across the cell and $H$ is the cell height, and the shear Reynolds number $\operatorname{Re}_{\tau}$,

$$
\operatorname{Re}_{\tau}=\frac{H}{2 v} \sqrt{\frac{\tilde{F} H}{2}} .
$$

We performed several runs with $\left(\mathrm{Ra}, \mathrm{Re}_{\tau}\right)$ within the ranges $\mathrm{Ra} \in\left[0 ; 1.3 \times 10^{7}\right]$ and $\operatorname{Re}_{\tau} \in[0 ; 205]$; the Prandtl number $\operatorname{Pr}=\nu / \kappa$ is kept fixed and equal to one.

A typical key question in RB studies is how the dimensionless heat flux through the cell, $\mathrm{Nu}$ [Eq. (3)], varies as a function

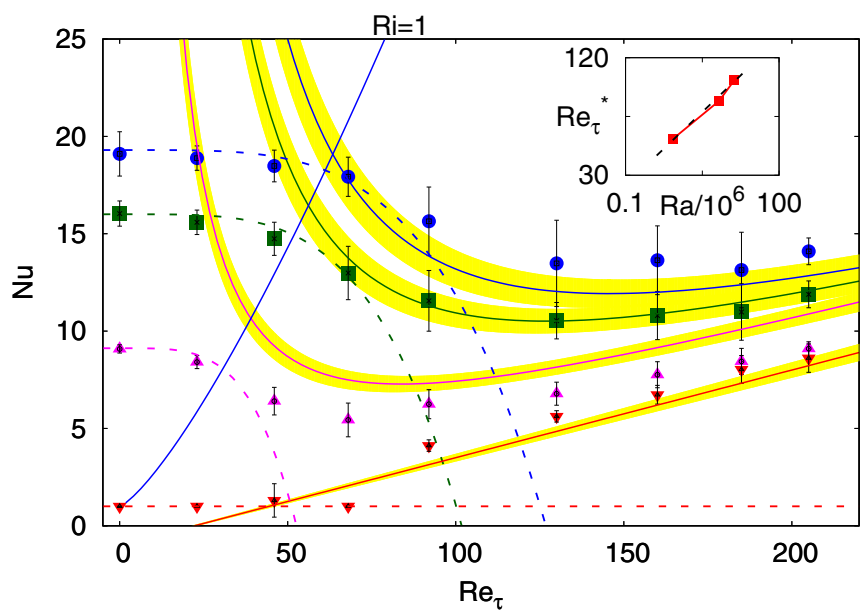

FIG. 2. (Color online) Nusselt number as a function of $\operatorname{Re}_{\tau}$ for $\mathrm{Ra}=0,8.125 \times 10^{5}, 6.5 \times 10^{6}, 1.3 \times 10^{7}$ (from bottom to top, symbols are, respectively, $\boldsymbol{\nabla}, \mathbf{\Lambda}, \mathbf{\square}, \bullet)$. Error bars are estimated from the fluctuations in time of the space average of $\mathrm{Nu}$ (for some cases uncertainties are smaller than the symbol size); typical integration times in the statistically steady state are $\sim 200 T_{L}$ (see Table I), with $T_{L}$ being the large eddy turnover time. Notice the nonmonotonic behavior for $\mathrm{Ra}>0$ : for low-to-moderate $\mathrm{Re}_{\tau}$ a decrease of $\mathrm{Nu}$ is observed due to plume sweeping by the mean wind; at higher $\operatorname{Re}_{\tau}$, when buoyancy is basically irrelevant with respect to the channel flow, $\mathrm{Nu}$ increases again, as expected for turbulent forced convection. The solid lines are the predictions from Eq. (16) obtained using $A_{1}=2.1$ and $A_{2}=0.045$, values that have been chosen to best fit the case $\mathrm{Ra}=6.5 \times 10^{6}$ ( $\mathbf{\square}$ symbols). The band around solid lines shows the prediction of the model for a variation of the parameters $A_{1}$ and $A_{2}$ of $\pm 10 \%$. The dashed lines represent the falloff for small $\operatorname{Re}_{\tau}$ provided by Eq. (21), with $A_{3}=1$ for all the three Ra; we also report the $\mathrm{Ri}=1$ curve, giving an estimate of the locus of points [in the ( $\mathrm{Ra}$, $\mathrm{Re}_{\tau}$ ) plane] where the crossover between the two regimes occurs. The reader is referred to the text for the details. Inset: Log-log plot of the shear Reynolds number $\mathrm{Re}_{\tau}^{*}$ corresponding to the crossover to the $\mathrm{Nu} \sim \mathrm{Re}_{\tau}^{-3 / 2}$ regime [23] as function of Rayleigh number. The dashed line is the $\operatorname{Re}_{\tau}^{*} \sim \mathrm{Ra}^{1 / 4}$ power law [Eq. (19)].

of the Rayleigh number, Ra:

$$
\mathrm{Nu}(z)=\frac{\overline{u_{z} T}(z)-\kappa \partial_{z} \bar{T}(z)}{\kappa \frac{\Delta}{H}}=\text { const. } \equiv \mathrm{Nu},
$$

here and hereafter the overline indicates a spatial (over planes $z=$ const.) and temporal (over the statistically stationary state) average. The second and third equalities (which state that $\mathrm{Nu}$ is constant with $z$ ) follow from taking the average of Eq. (2). In our setup in addition to buoyancy there is the longitudinal pressure gradient which affects the heat flux. We therefore focus on the dependence of $\mathrm{Nu}$ on the two-dimensional parameter space $\left(\mathrm{Ra}_{\mathrm{T}} \mathrm{Re}_{\tau}\right)$; in Fig. 2 we plot $\mathrm{Nu}$ as a function of $\mathrm{Re}_{\tau}$ for various fixed $\mathrm{Ra}$. We find that, for moderate and high $\mathrm{Ra}$, the effect of the lateral wind is to quench the buoyancy driven convection, and thus $\mathrm{Nu}$ decreases with $\mathrm{Re}_{\tau}$. For very low $\mathrm{Ra}$, below the critical Rayleigh number $\mathrm{Ra}_{c}$, the dynamics of the flow is instead completely dominated by the forced convection and thus $\mathrm{Nu}$ increases with $\mathrm{Re}_{\tau}$ (in Fig. 2 we show also the $\mathrm{Ra}=0$ case). Correspondingly, at increasing $\operatorname{Re}_{\tau}$ the 
TABLE I. Averaging times $T_{\text {avg }}$ (in units of the large eddy turnover time $T_{L}$ ) and relative error $\delta \mathrm{Nu} / \mathrm{Nu}$ in the computation of the Nusselt number for six cases from Fig. 2.

\begin{tabular}{lccc}
\hline \hline $\mathrm{Ra}$ & $\operatorname{Re}_{\tau}$ & $T_{\text {avg }}$ & $100 \times \delta \mathrm{Nu} / \mathrm{Nu}$ \\
\hline $1.3 \times 10^{7}$ & 0 & $225 T_{L}$ & $12 \%$ \\
$1.3 \times 10^{7}$ & 205 & $300 T_{L}$ & $9.7 \%$ \\
$6.5 \times 10^{6}$ & 0 & $180 T_{L}$ & $8 \%$ \\
$6.5 \times 10^{6}$ & 205 & $240 T_{L}$ & $11.5 \%$ \\
$8.125 \times 10^{5}$ & 0 & $65 T_{L}$ & $5 \%$ \\
$8.125 \times 10^{5}$ & 205 & $250 T_{L}$ & $7.7 \%$ \\
\hline \hline
\end{tabular}

mean temperature profiles (see Fig. 3) show a bending in the bulk and a decrease of the gradient in the boundary layer.

Our interpretation of these observations is that, for small $\mathrm{Re}_{\tau}$ or high $\mathrm{Ra}$ (i.e., in the natural convection dominated regime), the wind essentially sweeps away the thermal plumes (which subsequently lose coherence due to mixing) and hence the heat flux is depleted. Increasing $\operatorname{Re}_{\tau}$ further, we eventually reach a state where buoyancy becomes irrelevant. Here, $\mathrm{Nu}$ starts to increase again by resuspension of temperature puffs in the bulk due to bursts from the wall emerging because of the turbulent channel flow. To give an indication of the validity of such a conjecture we have measured the following quantity:

$$
\phi_{\ell}(z) \stackrel{\text { def }}{=} \frac{\overline{\left(\delta_{\ell} u_{z}\right)^{2}}}{\overline{\left(\delta_{\ell} u_{x}\right)^{2}}},
$$

where $\delta_{\ell} u_{i} \equiv u_{i}(x+\ell, y, z ; t)-u_{i}(x, y, z ; t)$. The observable (4) is the ratio of a generalized second-order transverse over longitudinal structure function and, as such, it serves as a scale-dependent anisotropy indicator: a large value of $\phi_{\ell}(z)$ means a coherent motion in the wall-normal direction. In Fig. 4 we plot $\phi_{\ell}(z)$ on a large scale $(\ell \approx H)$ and on a scale of the order of the thermal boundary layer thickness $\left(\ell=\lambda_{\theta}\right)$ of the pure $\mathrm{RB}$ case $\left(\operatorname{Re}_{\tau}=0\right)$, which gives an estimate of

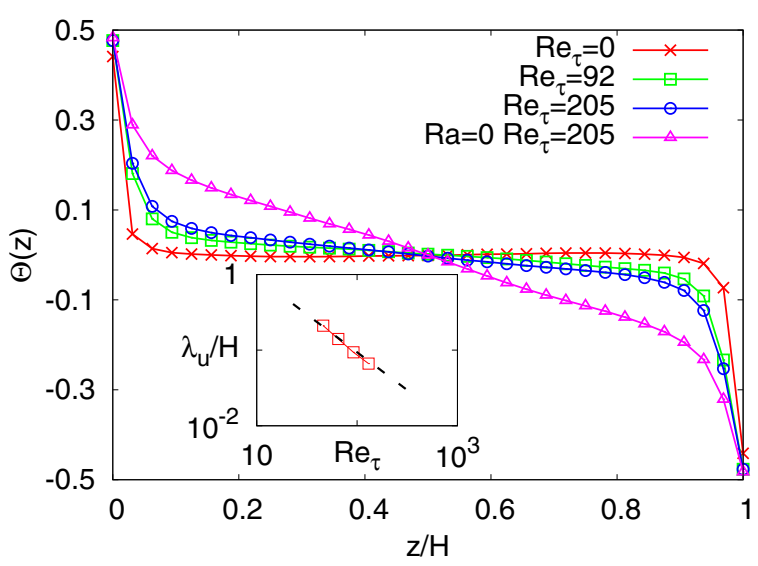

FIG. 3. (Color online) Normalized temperature profiles $\Theta(z) \equiv$ $\frac{\bar{T}(z)-T_{m}}{\Delta} \quad\left(T_{m}=\left(T_{\text {hot }}+T_{\text {cold }}\right) / 2\right.$ being the mean temperature $)$ for various $\operatorname{Re}_{\tau}$ at a fixed $\mathrm{Ra}=6.5 \times 10^{6}$. Notice the bending of the profile in the bulk in comparison with the usual "thermal shortcut" for $\operatorname{Re}_{\tau}=0$ (i.e., no lateral wind). Inset: Kinetic boundary layer thickness $\lambda_{u} \mathrm{vs}_{\tau}$ (log-log scale) for $\mathrm{Ra}=0$ : numerical data (symbols) and $\operatorname{Re}_{\tau}^{-1}$ scaling (dashed line).

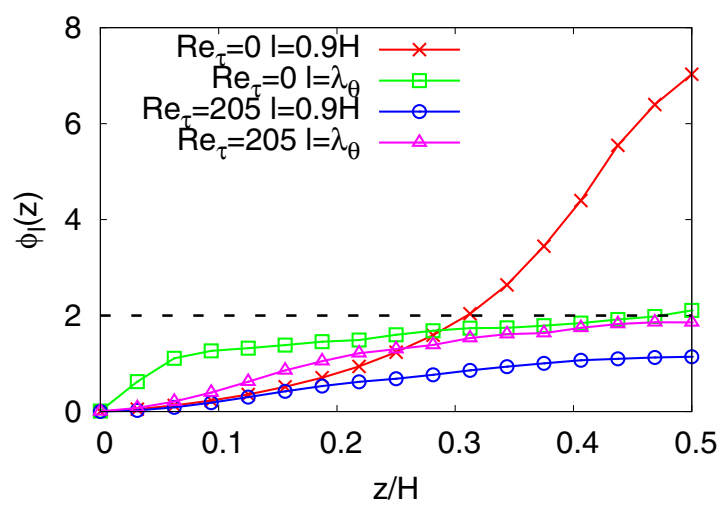

FIG. 4. (Color online) The anisotropy indicator defined in Eq. (4) as function of the cell height for pure $\operatorname{RB}\left(\operatorname{Re}_{\tau}=0\right)$ and for $\operatorname{Re}_{\tau}=205$ and for two separations in $x$, at $\mathrm{Ra}=6.5 \times 10^{6}$.

the characteristic size of "unperturbed" plumes, for natural convection $\left(\mathrm{Ra}=6.5 \times 10^{6}, \mathrm{Re}_{\tau}=0\right)$ and for a case with the wind $\left(\mathrm{Ra}=6.5 \times 10^{6}, \mathrm{Re}_{\tau}=205\right)$.

For the pure RB case $\phi_{\ell \approx H}(z)$ grows to large values in the bulk, due to the thermal wind, while $\phi_{\ell=\lambda_{\theta}}(z)$ goes to the isotropic value $\phi \approx 2$ in the bulk and it is larger than $\phi_{\ell \approx H}(z)$ close to the wall, pointing out the presence of detaching plumes. The same quantity $\phi_{\ell=\lambda_{\theta}}(z)$ in the wall-proximal region is significantly smaller for $\operatorname{Re}_{\tau}=205$, indicating the depletion of plume ejection.

\section{PHENOMENOLOGICAL MODELLING}

With this picture in mind we are now going to build a model to recover the numerical findings. Our argument goes as follows: As shown in Fig. 3, under the action of the lateral wind the temperature profile ceases to be flat in the bulk. This permits us to write a first-order closure for the turbulent heat flux of the kind

$$
\overline{u_{z} T}=-\kappa_{T} \partial_{z} \bar{T},
$$

where $\kappa_{T}$ is a turbulent diffusivity. When writing Eq. (5), where $\kappa_{T}$ is constant with $z$, we are implicitly restricting ourselves to the bulk region (where the mean temperature gradient is basically constant); we are allowed to do that by Eq. (3), i.e., the constancy of the heat flux through planes parallel to the walls. The Nusselt number will assume the form

$$
\mathrm{Nu} \sim\left(1+\frac{\kappa_{T}}{\kappa}\right) \frac{\left|\partial_{z} \bar{T}\right|}{(\Delta / H)} .
$$

We consider that two types of structures contribute to turbulent diffusion; namely, buoyant plumes $\left(\kappa_{T}^{(P)}\right)$ and bursts $\left(\kappa_{T}^{(B)}\right.$, triggered by the turbulent channel flow), so that we may write

$$
\kappa_{T}=\kappa_{T}^{(P)}+\kappa_{T}^{(B)} .
$$

As previously discussed we ascribe the heat flux depletion to the sweeping of plumes by the wind; we model this by assuming that the plume, being distorted by the strong shear close to the boundaries, loses its coherence (and, hence, it releases its heat content) after travelling a distance from the wall of the order of the kinetic boundary layer thickness $\left(\lambda_{u}\right)$, 
i.e., we suggest that we can adopt a Prandtl mixing length $\left(\ell_{m}\right)$ theory approach, using

$$
\ell_{m} \sim \lambda_{u}
$$

the latter relation should be interpreted as a scaling (or proportionality) relation rather than an order of magnitude. The characteristic velocity of a rising plume reaching a height $\sim \ell_{m}$ can be estimated as $u \sim \sqrt{\alpha g \Delta \ell_{m}}$ [24]; hence the contribution to the turbulent diffusion will be

$$
\kappa_{T}^{(P)}=\sqrt{\alpha g \Delta} \lambda_{u}^{3 / 2}
$$

and assuming a laminar boundary layer of Blasius type of thickness [25]

$$
\lambda_{u} \sim \frac{H}{\operatorname{Re}_{\tau}}
$$

we get

$$
\kappa_{T}^{(P)} \sim \sqrt{\alpha g \Delta} \frac{H^{3 / 2}}{\operatorname{Re}_{\tau}^{3 / 2}} .
$$

The validity of the effective scaling (10) has been checked a posteriori in the simulations, as can be seen in the inset of Fig. 3. For a turbulent burst one can also assume that $\ell_{m} \sim \lambda_{u}$, but the expression for the characteristic advecting velocity requires some more care. In a pure forced convection setup (or in our case when the wind is dominant) there is no buoyancy, so we cannot use the expression of the free-fall velocity; instead convection is driven by turbulent fluctuations from the wall. Invoking again the mixing length theory for a first-order closure for the velocity we can write $u_{z} \sim \ell_{m} \partial_{z} \bar{U}_{x}$, whence

$$
\kappa_{T}^{(B)} \sim \ell_{m}^{2} \partial_{z} \bar{U}_{x} \sim \lambda_{u}^{2} \partial_{z} \bar{U}_{x}
$$

estimating the shear as $\partial_{z} U_{x} \sim U_{c} / \lambda_{u}$ ( $U_{c}$ being the centerline velocity) we get

$$
\kappa_{T}^{(B)} \sim \lambda_{u} U_{c} .
$$

In the limited range of $\operatorname{Re}_{\tau}$ that we span it is reasonable to assume that the friction coefficient goes as $C_{f} \sim \operatorname{Re}_{\tau}^{-2}$, hence that $U_{c}$ scale as $U_{c} \sim(v / H) \operatorname{Re}_{\tau}^{2}$ [26]. Inserting this scaling law together with the relation (10) inside the expression for $\kappa_{T}^{(B)}$ we obtain

$$
\kappa_{T}^{(B)} \sim \nu \operatorname{Re}_{\tau} .
$$

Putting the expressions (11) and (14) inside Eq. (6) we end up with

$$
\mathrm{Nu}-1 \sim\left(A_{1} \frac{\sqrt{\alpha g \Delta} H^{3 / 2}}{\kappa \operatorname{Re}_{\tau}^{3 / 2}}+A_{2} \frac{\nu}{\kappa} \operatorname{Re}_{\tau}\right) \frac{\left|\partial_{z} \bar{T}\right|}{(\Delta / H)},
$$

which can be recast, introducing the dimensionless numbers $\mathrm{Ra}$ and Pr, into the following form:

$$
\mathrm{Nu}-1 \sim\left(A_{1} \frac{\operatorname{Ra}^{1 / 2} \operatorname{Pr}^{1 / 2}}{\operatorname{Re}_{\tau}^{3 / 2}}+A_{2} \operatorname{PrRe}_{\tau}\right),
$$

where $A_{1}$ and $A_{2}$ are the two free parameters of the model [27]. Some comments on Eq. (16) are in order. First, it reproduces the nonmonotonic dependence of the heat flux, $\mathrm{Nu}$, on the applied wind, $\operatorname{Re}_{\tau}$, and it turns out to be in fair agreement with the numerical data (see Fig. 2). Second, it provides an argument for the scaling $\mathrm{Nu} \sim \mathrm{Re}_{\tau}$ for the case of pure forced convection $(\mathrm{Ra}=0$, see Fig. 2). It is interesting to note that, for very small $\operatorname{Re}_{\tau}$, our model would give a scaling $\mathrm{Nu} \sim \mathrm{Ra}^{1 / 2}$, i.e., as expected in Kraichnan's ultimate regime of convection [2]. The phenomenology behind it suggests that the lower heat flux observed (with respect to $\mathrm{Ra}^{1 / 2}$ ) is a result of a negative feedback of the shear, due to the large scale circulation, on the plumes detaching from the boundary layer. Indeed, if we imagine the Nusselt number to follow a Kraichnan scaling on an effective Rayleigh $\mathrm{Ra}_{\mathrm{eff}}$, renormalized by turbulent viscosity and thermal diffusivity [behaving as Eq. (14)], that is,

$$
\mathrm{Ra}_{\mathrm{eff}}=\frac{\mathrm{Ra}}{\left(\nu_{T} / \nu\right)\left(\kappa_{T} / \kappa\right)},
$$

we end up with the following relation:

$$
\mathrm{Nu} \sim \mathrm{Ra}_{\mathrm{eff}}^{1 / 2} \equiv \frac{\mathrm{Ra}^{1 / 2}}{\mathrm{Re}_{\tau}} .
$$

If we now insert into Eq. (17) the ultimate regime scaling for Reynolds $\operatorname{Re}_{\tau} \sim \mathrm{Ra}^{1 / 4}$ [28], we obtain

$$
\mathrm{Nu} \sim \mathrm{Ra}^{1 / 4}
$$

a well-known scaling, predicted theoretically and found in a vast number of experiments (see Ref. [11] and references therein). Let us, finally, remark that Eq. (16) should not be expected to be valid for $\operatorname{Re}_{\tau} \rightarrow 0$, since in this case the mean temperature gradient is zero and a closure like Eq. (5) does not apply [27]. In particular we detect a region where the sweeping mechanism is not yet effective and $\mathrm{Nu}$ decreases slowly with $\operatorname{Re}_{\tau}$; we denote the shear Reynolds number at which the crossover between such a region and the $\mathrm{Nu} \sim \mathrm{Re}_{\tau}^{-3 / 2}$ regime takes place as $\mathrm{Re}_{\tau}^{*}$, and we argue that such crossover can be determined under the condition that the characteristic velocity of a rising plume, $U^{(\mathrm{RB})} \sim \sqrt{\alpha g \Delta H}$, be of the same order of the centerline velocity of the Poiseuille flow, $U^{(P)} \sim \tilde{F} H^{2} / \nu$. Equating these two latter relations we have

$$
\sqrt{\alpha g \Delta H} \sim \frac{\tilde{F} H^{2}}{v},
$$

which gives, in dimensionless form and introducing the crossover Reynolds,

$$
\operatorname{Re}_{\tau}^{*} \sim \operatorname{Ra}^{1 / 4}
$$

This result is compared with the numerical data in the inset of Fig. 2. It is worth noticing that the condition (19) corresponds to the Richardson number $\mathrm{Ri}=\mathrm{Ra} / \mathrm{Re}_{\tau}^{4}$ (for $\mathrm{Pr}=1$ ) being of order unity (see the $\mathrm{Ri}=1$ curve in Fig. 2). For $\operatorname{Re}_{\tau}<\operatorname{Re}_{\tau}^{*}$ the longitudinal flow represents just a small disturbance to the buoyant circulation (notice that the Nusselt number for $\mathrm{Ra}=0$ remains equal to one). The initial falloff of $\mathrm{Nu} v \mathrm{Re}_{\tau}$ can be captured by looking at the conservation equation for the total energy, which can be derived from Eqs. (1) and (2) to be [10]

$$
\varepsilon=(\mathrm{Nu}-1) \mathrm{Ra}+8 \operatorname{Re}_{\tau}^{2}\left\langle u_{x}\right\rangle,
$$

where $\langle\cdots\rangle$ denotes an average over the entire volume, $\varepsilon=\left\langle\left(\partial_{i} u_{j}\right)^{2}\right\rangle$ is the kinetic energy dissipation rate (expressed in terms of nondimensionalized variables as in [10]), and we set $\operatorname{Pr}=1$. Denoting with the superscripts $(\mathrm{RB})$ and $(P)$ quantities relative to buoyancy (Rayleigh-Bénard) and pressure (Poiseuille) driven sectors, respectively, it is easy to see 
that $\left\langle u_{x}\right\rangle \equiv\left\langle\left(u_{x}^{(\mathrm{RB})}+u_{x}^{(P)}\right)\right\rangle \sim U^{(P)} \sim \operatorname{Re}_{\tau}^{2}$ [being $\left\langle u_{x}^{(\mathrm{RB})}\right\rangle \sim$ 0 valid due to incompressibility and to periodic boundary conditions in $(x, y)]$. Since $U^{(\mathrm{RB})} \gg U^{(P)}$ the longitudinal wind perturbs the RB dynamics only slightly so that $\varepsilon \approx$ $\varepsilon^{(\mathrm{RB})}$ [29]. From Eq. (20) we therefore derive

$$
\mathrm{Nu} \approx \mathrm{Nu}_{0}(\mathrm{Ra})-\left(A_{3} / \mathrm{Ra}\right) \mathrm{Re}_{\tau}^{4}
$$

where $\mathrm{Nu}_{0}$ is the Nusselt number for $\mathrm{Re}_{\tau}=0$, i.e., pure $\mathrm{RB}$, and $A_{3}$ is an order one constant. Equation (21) is plotted in Fig. 2 for three different Ra showing good agreement with the numerics up to the expected crossover shear Reynolds $\operatorname{Re}_{\tau}^{*}$.

\section{CONCLUSIONS}

We have performed direct numerical simulation of Rayleigh-Bénard convection with an imposed longitudinal pressure gradient inducing a mean wind. We found that the Nusselt number has a nonmonotonic dependence on the shear Reynolds number based on the applied pressure drop: to an initial decrease (justifiable in terms of a mechanism of sweeping of plumes by the longitudinal wind) an increase follows, when the dynamics are dominated by the turbulent "forced convection" regime. Based on these empirical concepts, we provided a correlation which recovers the numerical findings with reasonable accuracy. The observations and the modeling above suggest that, in standard RB convection, the shear due to the large scale circulation may act back onto the boundary layer against the ejection of plumes to the bulk (thus being a possible mechanism for the depletion of heat transfer respect to the ultimate state of turbulent convection). Our work is a first attempt to look directly at the effect of disturbing in a controlled manner the dynamics of the boundary layer in such a way to give an insight of its role in natural convection. A possible followup of the present study is to use a perturbation other than a simple Poiseuille flow.

\section{ACKNOWLEDGMENTS}

We thank R. Benzi, P. Roche, R. P. J. Kunnen, F. Zonta, and P. Ripesi for useful discussions and L. Bouhlali and $H$. Einarsson for a careful reading of the manuscript and for their help with some analysis. A.S. and A.G. acknowledge financial support from the Icelandic Research Fund. A.S. acknowledges the Department of Mathematics and Computer Science of the Eindhoven University of Technology for the hospitality.
[1] L. P. Kadanoff, Phys. Today 54(8), 34 (2001).

[2] G. Ahlers, S. Grossmann, and D. Lohse, Rev. Mod. Phys. 81, 503 (2009).

[3] D. Lohse and K.-Q. Xia, Annu. Rev. Fluid Mech. 42, 335 (2010).

[4] J. Schumacher and F. Chillà, Eur. Phys. J. E 35, 58 (2012).

[5] H. B. Bluestein, Severe Convective Storms and Tornadoes (Springer, Berlin, Heidelberg, 2013).

[6] J. J. M. Sillekens, C. C. M. Rindt, and A. A. Van Steenhoven, Int. J. Heat Mass Transfer 41, 61 (1998).

[7] P. F. Linden, Annu. Rev. Fluid Mech. 31, 201 (1999).

[8] J. Bailon-Cuba, O. Shishkina, C. Wagner, and J. Schumacher, Phys. Fluids 24, 107101 (2012).

[9] O. Shishkina and C. Wagner, J. Turbul. 13, N22 (2012).

[10] B. I. Shraiman and E. D. Siggia, Phys. Rev. A 42, 3650 (1990).

[11] S. Grossmann and D. Lohse, J. Fluid Mech. 407, 27 (2000).

[12] S. Grossmann and D. Lohse, Phys. Fluids 16, 4462 (2004).

[13] R. J. A. M. Stevens, E. P. van der Poel, S. Grossmann, and D. Lohse, J. Fluid Mech. 730, 295 (2013).

[14] K. S. Gage and W. H. Reid, J. Fluid Mech. 33, 21 (1968).

[15] P. Carrière, P. A. Monkewitz, and D. Martinand, J. Fluid Mech. 502, 153 (2004).

[16] F. Zonta and A. Soldati, J. Heat Transfer 136, 022501 (2014).

[17] J. A. Domaradzki and R. W. Metcalfe, J. Fluid Mech. 193, 499 (1988).

[18] S. Succi, The Lattice Boltzmann Equation for Fluid Dynamics and Beyond (Oxford University Press, Oxford, 2001).

[19] D. Wolf-Gladrow, Lattice Gas Cellular Automata and Lattice Boltzmann Methods (Springer, Berlin, Heidelberg, 2000).
[20] R. Benzi, F. Toschi, and R. Tripiccione, J. Stat. Phys. 93, 901 (1998); E. Calzavarini, F. Toschi, and R. Tripiccione, Phys. Rev. E 66, 016304 (2002).

[21] F. Toschi, G. Amati, S. Succi, R. Benzi, and R. Piva, Phys. Rev. Lett. 82, 5044 (1999); R. Benzi et al., Phys. Fluids 11, 1284 (1999); F. Toschi, E. Lévêque, and G. Ruiz-Chavarría, Phys. Rev. Lett. 85, 1436 (2000); L. Biferale et al., J. Fluid Mech. 452, 39 (2002).

[22] V. Lavezzo, H. J. H. Clercx, and F. Toschi, J. Phys.: Conf. Ser. 333, 012011 (2011).

[23] We estimate $\mathrm{Re}_{\tau}^{*}$ from the intersection of the best fit of Eqs. (16) and (21) to the numerical data.

[24] We assume that the acceleration experienced by the plume is proportional to the temperature difference $\Delta$, i.e., that the bending of the thermal shortcut is small.

[25] L. D. Landau and E. M. Lifshitz, Fluid Mechanics, 536 (Pergamon, New York, 1959).

[26] More precisely our simulations suggest something closer to $U_{c} \sim \operatorname{Re}_{\tau}^{1.9}$.

[27] In principle there would be an extra dependence of $\mathrm{Nu}$ on $\mathrm{Re}_{\tau}$ in Eq. (16) stemming from $\left|\partial_{z} \bar{T}\right| /(\Delta / H) \sim f\left(\operatorname{Re}_{\tau}\right)$ which, however, turns out to be a subdominant correction, only becoming relevant for very low $\mathrm{Re}_{\tau}$; we assume, then, $f\left(\mathrm{Re}_{\tau}\right) \approx$ const. and absorb it into the two parameters $A_{1}, A_{2}$. K. Hanjalic, Annu. Rev. Fluid Mech. 34, 321 (2002).

[28] The scaling is $\operatorname{Re} \sim \operatorname{Ra}^{1 / 2}$ and then, since $\operatorname{Re}_{\tau} \sim \operatorname{Re}^{1 / 2}$, we get $\mathrm{Re}_{\tau} \sim \mathrm{Ra}^{1 / 4}$.

[29] We assume that the energy dissipation rate is not affected by the longitudinal wind, at least in its boundary layer contributions which are dominant in this regime (our numerical simulations confirm this picture). 